ANNALES

POLONICI MATHEMATICI

$92.1(2007)$

\title{
An example of a pseudoconvex domain whose holomorphic sectional curvature of the Bergman metric is unbounded
}

\author{
by Gregor Herbort (Wuppertal)
}

\begin{abstract}
Let $a$ and $m$ be positive integers such that $2 a<m$. We show that in the domain $D:=\left\{\left.z \in \mathbb{C}^{3}\left|r(z):=\operatorname{Re} z_{1}+\right| z_{1}\right|^{2}+\left|z_{2}\right|^{2 m}+\left|z_{2} z_{3}\right|^{2 a}+\left|z_{3}\right|^{2 m}<0\right\}$ the holomorphic sectional curvature $R_{D}(z ; X)$ of the Bergman metric at $z$ in direction $X$ tends to $-\infty$ when $z$ tends to 0 non-tangentially, and the direction $X$ is suitably chosen. It seems that an example with this feature has not been known so far.
\end{abstract}

1. Introduction. The unit disc, equipped with the Poincaré metric, is a first example of a domain with a metric of constant negative curvature. The generalization to the unit ball in $\mathbb{C}^{n}$ with $n \geq 2$ is given by the Bergman metric. Its holomorphic sectional curvature $R$ is also a negative constant. By a result of Lu Qi-Keng [17] the ball is the only simply connected domain (up to biholomorphic equivalence) whose Bergman metric has negative constant holomorphic curvature (see also [9]).

Since the results of [5] and [12] it has become possible to determine, on a bounded strongly pseudoconvex domain $D$, the boundary behavior of the holomorphic sectional curvature $R_{D}(z ; X)$ for $(z, X) \in D \times \mathbb{C}^{n}$. For a $C^{\infty}$ smooth strongly pseudoconvex domain Klembeck [15] has shown, by means of the Fefferman asymptotic formula for the Bergman kernel function, that for any tangent vector $X \neq 0$ the quantity $R_{D}(z ; X)$ tends to $-2 /(n+1)$ when $z$ tends to the boundary. His smoothness assumption was considerably weakened later in [14].

Since the investigations of Bergman [1] it has been known that the holomorphic sectional curvature of the Bergman metric is always less than 2 . That it is bounded from below is known in the class of strongly pseudoconvex domains (this is obvious) and also (by [18]) in smooth bounded pseudoconvex domains of finite type in $\mathbb{C}^{2}$. In [8] the case of smoothly bounded

2000 Mathematics Subject Classification: 32F45, 32T25.

Key words and phrases: Bergman metric, holomorphic sectional curvature. 
Reinhardt domains of finite type in $\mathbb{C}^{2}$ was treated. The holomorphic sectional curvature of the Bergman metric in such domains, in a neighborhood of the boundary, can be estimated from above by a negative constant.

In the present note we give an example of a smooth bounded pseudoconvex Reinhardt domain $D$ of finite type in $\mathbb{C}^{3}$ such that the holomorphic sectional curvature $R_{D}(z ; X)$ of the Bergman metric is not bounded from below in certain directions $X$. The idea and the kind of argument used are completely in the spirit of $[10,11]$ (see also [7]).

TheOREM 1.1. Let $a, m$ be positive integers such that $2 a<m$. Let $D:=\left\{\left.z \in \mathbb{C}^{3}\left|r(z):=\operatorname{Re} z_{1}+\right| z_{1}\right|^{2}+P\left(z^{\prime}\right)<0\right\}$, where $P\left(z^{\prime}\right):=P\left(z_{2}, z_{3}\right)$ $:=\left|z_{2}\right|^{2 m}+\left|z_{2} z_{3}\right|^{2 a}+\left|z_{3}\right|^{2 m}$. Then the holomorphic sectional curvature $R_{D}\left(-\right.$ te $\left._{1} ; X\right)$ tends to $-\infty$ as $t \searrow 0$ if $X=\left(0, X^{\prime}\right) \in\{0\} \times \mathbb{C}^{2}$ and $X^{\prime}=\left(X_{2}, X_{3}\right)$ with $X_{2}, X_{3} \neq 0$. Here $e_{1}=(1,0,0)$. More precisely,

$$
2-R_{D}\left(-t e_{1} ;\left(0, X^{\prime}\right)\right) \approx \frac{1}{\log (1 / t)}\left(1+\frac{1}{t^{1 / a-2 / m} \log (1 / t)} \frac{\left|X_{2}\right|^{2}\left|X_{3}\right|^{2}}{\left|X^{\prime}\right|^{4}}\right) .
$$

The above domain is a Reinhardt domain with center at $\zeta_{0}=-\frac{1}{2} e_{1}$. A phenomenon as described in the theorem is not possible in domains all of whose boundary points are of finite semiregular type (see [2] or [16]). The notion of semiregular type was defined in [6] (see also [19]). A point $\zeta$ in a smooth hypersurface $M$ is said to be of (finite) semiregular type if the D'Angelo type $\Delta_{1}(\zeta, M)$ of $M$ at $\zeta$ is finite, and the $n$-tuple $\left(1, \Delta_{n-1}(\zeta, M)\right.$, $\left.\ldots, \Delta_{1}(\zeta, M)\right)$ of the D'Angelo higher type numbers equals the Catlin multitype $\left(1, m_{2}, \ldots, m_{n}\right)$ of $M$ at $\zeta$. In dimension 3 a point $\zeta \in M$ is of semiregular type if the D'Angelo type at $\zeta$ is finite and equal to the entry $m_{3}$ of the Catlin multitype.

In our domain the assumption $2 a<m$ implies $1 / a-2 / m>0$. It prevents the origin from being a point of (finite) semiregular type. Indeed, the Catlin multitype of $\partial D$ at 0 is $(1,4 a, 4 a)$, while the D'Angelo type at this point is $2 m$.

Stimulation for this article came from the paper [4], where for the first time an example of a domain $\Omega$ was given in which the holomorphic sectional curvature $R_{\Omega}(z ; X)$ of the Bergman metric tends to 2 as $z$ tends to a certain boundary point of $D$ and the direction $X$ is suitably chosen. Also, in [4] it was asked whether there exists a bounded pseudoconvex domain whose holomorphic sectional curvature with respect to the Bergman metric is unbounded.

Acknowledgements. I would like to thank the referee for checking my manuscript so carefully, and for valuable suggestions on the preparation of the corrected version. 


\section{Proof of Theorem 1.1}

The relevant domain functionals. Let $\Omega \subset \subset \mathbb{C}^{n}$ be a bounded domain. We denote by $H^{2}(\Omega)$ the Hilbert space of all holomorphic functions on $\Omega$ that are square-integrable with respect to the Lebesgue measure. Put

$$
\|f\|_{\Omega}^{2}:=\int_{\Omega}|f|^{2} d^{2 n} z
$$

For $z \in \Omega$ we consider the following subsets of $H^{2}(\Omega)$ :

$$
\begin{aligned}
\mathcal{A}_{0}(\Omega) & :=\left\{f \in H^{2}(\Omega) \mid\|f\|_{\Omega} \leq 1\right\}, \\
\mathcal{A}_{1}(\Omega ; z) & :=\left\{f \in \mathcal{A}_{0}(\Omega) \mid f(z)=0\right\}, \\
\mathcal{A}_{2}(\Omega ; z) & :=\left\{f \in \mathcal{A}_{1}(\Omega) \mid \frac{\partial f(z)}{\partial z_{j}}=0, j=1, \ldots, n\right\} .
\end{aligned}
$$

Then we have the well-known relationships between the Bergman kernel $K_{\Omega}: \Omega \rightarrow \mathbb{R}$, the Bergman metric $B_{\Omega}^{2}(z ; X)$ at $z$ in direction $X$, and the holomorphic sectional curvature $R_{\Omega}(z ; X)$ of the Bergman metric for $(z, X) \in \Omega \times \mathbb{C}^{n}:$

$$
\begin{gathered}
K_{\Omega}(z)=\sup \left\{|f(z)|^{2} \mid f \in \mathcal{A}_{0}(\Omega)\right\}, \\
B_{\Omega}^{2}(z ; X)=\frac{J_{1, \Omega}(z ; X)}{K_{\Omega}(z)}, \\
2-R_{\Omega}(z ; X)=\frac{K_{\Omega}(z) J_{2, \Omega}(z ; X)}{J_{1, \Omega}(z ; X)^{2}},
\end{gathered}
$$

where

$$
\begin{aligned}
& J_{1, \Omega}(z ; X):=\sup \left\{|X(f)(z)|^{2} \mid f \in \mathcal{A}_{1}(\Omega ; z)\right\}, \\
& J_{2, \Omega}(z ; X):=\sup \left\{|X X(f)(z)|^{2} \mid f \in \mathcal{A}_{2}(\Omega ; z)\right\} .
\end{aligned}
$$

For a vector $X:=\left(X_{1}, \ldots, X_{n}\right) \in \mathbb{C}^{n}$ we denote by $X(f)(z)$ the derivative of $f$ at $z$ in direction $X$, explicitly $X(f)(z):=\sum_{j=1}^{n} \frac{\partial f(z)}{\partial z_{j}} X_{j}$, and $X X(f)(z)=$ $\sum_{j, k=1}^{n} \frac{\partial^{2} f(z)}{\partial z_{j} \partial z_{k}} X_{j} X_{k}$.

Splitting off the $z_{1}$-direction. For $s>0$ we put $D_{s}:=\left\{z^{\prime} \in \mathbb{C}^{2} \mid P\left(z^{\prime}\right)<s\right\}$. Note that for $0<t<1 / 10$ the domain

$$
\widetilde{D}_{t}:=\Delta\left(-t, \frac{t-t^{2}}{2}\right) \times D_{\left(t-t^{2}\right) / 4}
$$

is contained in $D$.

The following comparison lemma is needed for the proof of Lemma 2.2 below. Its proof is based on a standard $\bar{\partial}$-argument. The idea is in the spirit of $[3$, Sec. 6$]$. 
Lemma 2.1. There exists a constant $C>0$ such that, for any $0<t<$ $1 / 10$ and any function $f \in H^{2}\left(\widetilde{D}_{t}\right)$, we can find a function $\widehat{f} \in H^{2}(D)$ such that $\|\widehat{f}\|_{D} \leq C\|f\|_{\widetilde{D}_{t}}$, and $\widehat{f}-f$ vanishes to third order at the point $-t e_{1}$.

Proof. STEP 1: We construct a plurisubharmonic weight function that fits well the size of $\widetilde{D}_{t}$.

First we note that

$$
-2 t \leq r(z) \leq-t / 4 \quad \text { on } \widetilde{D}_{t}
$$

Let

$$
W_{t}(z):=\frac{\left|z_{1}+t\right|^{2}}{t^{2}}+\frac{1}{t} P\left(z^{\prime}\right) .
$$

If now $z \in \mathbb{C}^{3}$ and $W_{t}(z) \leq 1 / 5$, then $\left|z_{1}+t\right|<t / \sqrt{5}<\left(t-t^{2}\right) / 2$ when $t<1 / 10$. Because also $P\left(z^{\prime}\right)<t / 5<\left(t-t^{2}\right) / 4$, we see that $z \in \widetilde{D}_{t}$. The Levi form of the plurisubharmonic function $\psi_{t}:=e^{r / t}$ can be estimated on the set $\left\{W_{t} \leq 1 / 5\right\}$ by

$$
\begin{aligned}
\mathscr{L}_{\psi_{t}}(z ; X) & =e^{r / t}\left(\frac{\left|X_{1}\right|^{2}}{t}+\frac{1}{t} \mathscr{L}_{P}\left(z^{\prime} ; X^{\prime}\right)+\left|\frac{\left(1+2 \bar{z}_{1}\right) X_{1}}{2 t}+\frac{\left\langle\partial P\left(z^{\prime}\right), X^{\prime}\right\rangle}{t}\right|^{2}\right) \\
& \geq e^{r / t}\left(\frac{1}{t} \mathscr{L}_{P}\left(z^{\prime} ; X^{\prime}\right)+\frac{\left|\left(1+2 \bar{z}_{1}\right) X_{1}\right|^{2}}{8 t^{2}}-2 \frac{\left|\left\langle\partial P\left(z^{\prime}\right), X^{\prime}\right\rangle\right|^{2}}{t^{2}}\right) \\
& \geq e^{r / t}\left(\frac{1}{t} \mathscr{L}_{P}\left(z^{\prime} ; X^{\prime}\right)+\frac{\left|(1-3 t) X_{1}\right|^{2}}{8 t^{2}}-2 \frac{\left|\left\langle\partial P\left(z^{\prime}\right), X^{\prime}\right\rangle\right|^{2}}{t^{2}}\right) \\
& \geq e^{r / t}\left(\frac{1}{t} \mathscr{L}_{P}\left(z^{\prime} ; X^{\prime}\right)+\frac{49\left|X_{1}\right|^{2}}{800 t^{2}}-2 \frac{P\left(z^{\prime}\right) \mathscr{L}_{P}\left(z^{\prime} ; X^{\prime}\right)}{t^{2}}\right) \\
& \geq e^{r / t}\left(\frac{1}{t}\left(1-2 \frac{P\left(z^{\prime}\right)}{t}\right) \mathscr{L}_{P}\left(z^{\prime} ; X^{\prime}\right)+0.06 \frac{\left|X_{1}\right|^{2}}{t^{2}}\right) \\
& \geq e^{-2}\left(0.06 \frac{\left|X_{1}\right|^{2}}{t^{2}}+\frac{3}{5 t} \mathscr{L}_{P}\left(z^{\prime} ; X^{\prime}\right)\right) \geq 0.06 e^{-2} \mathscr{L}_{W_{t}}(z ; X) .
\end{aligned}
$$

The estimate $\left|\left\langle\partial P\left(z^{\prime}\right), X^{\prime}\right\rangle\right|^{2} \leq P\left(z^{\prime}\right) \mathscr{L}_{P}\left(z^{\prime} ; X^{\prime}\right)$ follows from the fact that $\log P$ is plurisubharmonic.

Next we choose an increasing smooth function $\chi: \mathbb{R} \rightarrow(-\infty, 1]$ with

$$
\chi(s)= \begin{cases}s & \text { for } s \leq 1 / 10, \\ 1 & \text { for } s \geq 3 / 20,\end{cases}
$$

and put $V_{t}:=M \psi_{t}+\log \chi \circ W_{t}$. It is possible to choose $M$ independently of $t$ in such a way that $V_{t}$ becomes plurisubharmonic throughout $D$, and moreover,

$$
\mathscr{L}_{V_{t}} \geq \gamma \mathscr{L}_{W_{t}}
$$

on the set $\{W \leq 1 / 5\}$ for some constant $\gamma>0$ that does not depend on $t$. 
To see this, we note that

$$
\begin{aligned}
\mathscr{L}_{V_{t}}(z ; X)= & M \mathscr{L}_{\psi_{t}}(z ; X)+(\log \chi)^{\prime} \circ W_{t} \cdot \mathscr{L}_{W_{t}}(z ; X) \\
& +(\log \chi)^{\prime \prime} \circ W_{t} \cdot\left|\left\langle\partial W_{t}(z), X\right\rangle\right|^{2}
\end{aligned}
$$

for $(z, X) \in D \times \mathbb{C}^{3}$.

There are three cases to be considered:

(i) If $W_{t}(z) \in[0,1 / 10]$, we have ( $\log W_{t}$ is p.s.h.)

$$
\begin{aligned}
\mathscr{L}_{V_{t}}(z ; X) & =M \mathscr{L}_{\psi_{t}}(z ; X)+\mathscr{L}_{\log W_{t}}(z ; X) \\
& \geq M \mathscr{L}_{\psi_{t}}(z ; X) \geq 0.06 e^{-2} M \mathscr{L}_{W_{t}}(z ; X) .
\end{aligned}
$$

(ii) If $1 / 5 \geq W_{t}(z) \geq 3 / 20$, we have

$$
\mathscr{L}_{V_{t}}(z ; X)=M \mathscr{L}_{\psi_{t}}(z ; X) \geq 0.06 e^{-2} M \mathscr{L}_{W_{t}}(z ; X) .
$$

(iii) Assume that $1 / 10 \leq W_{t}(z) \leq 3 / 20$. With some constant $C_{3}>0$, we can estimate $(\log \chi)^{\prime}(s),(\log \chi)^{\prime \prime}(s) \geq-C_{3}$ for $s \in[1 / 10,3 / 20]$. This in conjunction with the log-plurisubharmonicity of $P$ gives

$$
\begin{aligned}
\mathscr{L}_{V_{t}}(z ; X) & \geq M \mathscr{L}_{\psi_{t}}(z ; X)-C_{3} \mathscr{L}_{W_{t}}(z ; X)-C_{3}\left|\left\langle\partial W_{t}(z), X\right\rangle\right|^{2} \\
& \geq M \mathscr{L}_{\psi_{t}}(z ; X)-C_{3}\left(1+W_{t}(z)\right) \mathscr{L}_{W_{t}}(z ; X) \\
& \geq M \mathscr{L}_{\psi_{t}}(z ; X)-2 C_{3} \mathscr{L}_{W_{t}}(z ; X) \\
& \geq\left(0.06 e^{-2} M-2 C_{3}\right) \mathscr{L}_{W_{t}}(z ; X) .
\end{aligned}
$$

We can now choose $M>200 C_{3} e^{2}$ and $\gamma=C_{3}$.

STEP 2: Let $\xi \in C^{\infty}(\mathbb{R})$ be a non-negative cut-off function satisfying $\xi(s)=1$ for $s \leq 1 / 10$ and $\chi(s)=0$ if $s \geq 1 / 5$. Given a function $f \in H^{2}\left(\widetilde{D}_{t}\right)$ we define the $\overline{\bar{\partial}}$-closed smooth $(0,1)$-form

$$
v=\bar{\partial}\left(\xi \circ W_{t}\right) \cdot f=\xi^{\prime} \circ W_{t} \cdot f \cdot \bar{\partial} W_{t} .
$$

This form is defined on $D$. Measuring its length with respect to the hermitian form $Q:=\mathscr{L}_{|z|^{2}+10 V_{t}}$, we will show that

$$
|v|_{Q}^{2} e^{-|z|^{2}-10 V_{t}} \leq C_{1}|f|^{2}
$$

with an unimportant constant $C_{1}>0$ (uniformly in $t$ ), where

$$
|v|_{Q}^{2}:=\sum_{j, k=1}^{n} Q^{j \bar{k}} v_{j} \bar{v}_{k}
$$

and $Q^{j \bar{k}}$ are the coefficients of the inverse of the matrix associated to $Q$ and the $v_{j}$ are the coefficients of $v$.

Namely, on $\operatorname{supp}(v) \subset\left\{1 / 10 \leq W_{t} \leq 1 / 5\right\}$ we have

and

$$
\mathscr{L}_{W_{t}} \geq \frac{1}{W_{t}} \partial W_{t} \otimes \bar{\partial} W_{t} \geq 5 \partial W_{t} \otimes \bar{\partial} W_{t}
$$

$$
Q=\mathscr{L}_{|z|^{2}}+10 \mathscr{L}_{V_{t}} \geq \mathscr{L}_{|z|^{2}}+10 \gamma \mathscr{L}_{W_{t}} \geq \mathscr{L}_{|z|^{2}}+50 \gamma \partial W_{t} \otimes \bar{\partial} W_{t}
$$


hence

$$
|v|_{Q}^{2}=\left(\xi^{\prime} \circ W_{t}\right)^{2}|f|^{2}\left|\bar{\partial} W_{t}\right|_{Q}^{2} \leq \frac{1}{50 \gamma}\left(\xi^{\prime} \circ W_{t}\right)^{2}|f|^{2} .
$$

Because of the monotonicity of $\chi$ the function $V_{t}$ is bounded from below on $\operatorname{supp}(v)$ by

$$
V_{t}=M \psi_{t}+\log \chi \circ W_{t} \geq \log (1 / 10) .
$$

This implies

$$
|v|_{Q}^{2} e^{-|z|^{2}-10 V_{t}} \leq \frac{10^{10}}{50 \gamma}\left(\xi^{\prime} \circ W_{t}\right)^{2}|f|^{2},
$$

which is $(*)$ with

$$
C_{1}=\frac{10^{10}}{50 \gamma} \max \left(\xi^{\prime} \circ W_{t}\right)^{2} .
$$

A refinement in the proof of Lemma 4.4.1 from [13] (which is by now standard) gives us a smooth solution $u$ to the equation $\bar{\partial} u=v$ that satisfies

$$
\int_{D}|u|^{2} e^{-|z|^{2}-10 V_{t}} d^{6} z \leq \int_{D}|v|_{Q}^{2} e^{-|z|^{2}-10 V_{t}} d^{6} z \leq C_{1}\|f\|_{\widetilde{D}_{t}}^{2} .
$$

Since $V_{t} \leq M$, we have $\int_{D}|u|^{2} d^{6} z \leq C_{1} e^{2(\operatorname{diam} D)^{2}+10 M}\|f\|_{\widetilde{D}_{t}}^{2}$, where $\operatorname{diam} D$ is the diameter of $D$. Furthermore, because of the term $10 V_{t}$ in the weight that appears on the left-hand side of $\left(L^{2}\right)$, the function $u$ vanishes to third order at the point $-t e_{1}$. Then the holomorphic function

$$
\widehat{f}:=\xi \circ W_{t} \cdot f-u
$$

satisfies $\|\widehat{f}\|_{L^{2}(D)} \leq C\|f\|_{\widetilde{D}_{t}}$, with $C:=1+\sqrt{C_{1}} e^{(\operatorname{diam} D)^{2}+5 M}$, and hence fulfills our requirements.

Lemma 2.1 is proved.

Corollary.

(i) For any function $f \in \mathcal{A}_{0}\left(\widetilde{D}_{t}\right)$ let $\widehat{f}$ be the function from Lemma 2.1 . Then $\widehat{f} / C \in \mathcal{A}_{0}(D)$, and

$$
K_{D}\left(-t e_{1}\right) \geq C^{-2} K_{\widetilde{D}_{t}}\left(-t e_{1}\right) .
$$

(ii) Likewise, for $k \in\{1,2\}$ and any function $f \in \mathcal{A}_{k}\left(\widetilde{D}_{t} ;-t e_{1}\right)$, the function $\widehat{f} / C$ belongs to the family $\mathcal{A}_{k}\left(D ;-t e_{1}\right)$, and for all $X \in \mathbb{C}^{3}$ we have

$$
J_{k, D}\left(-t e_{1} ; X\right) \geq C^{-2} J_{k, \widetilde{D}_{t}}\left(-t e_{1} ; X\right), \quad k=1,2 .
$$

This enables us to give lower and upper estimates for the domain functionals in formulas (a), (b), and (c) at the point $-t e_{1}$ by the corresponding domain functionals (evaluated at $0^{\prime}$ ) that belong to the domains $D_{t-t^{2}}$. 
Lemma 2.2. With a suitable constant $C_{*}>0$, for $0<t<1 / 10$ we have

$$
\frac{1}{C_{*} t^{2}} K_{D_{\left(t-t^{2}\right) / 4}}\left(0^{\prime}\right) \leq K_{D}\left(-t e_{1}\right) \leq \frac{5}{\pi t^{2}} K_{D_{\left(t-t^{2}\right) / 4}}\left(0^{\prime}\right),
$$

and for $X^{\prime} \in \mathbb{C}^{2}$ and $k=1,2$ we have

$$
\frac{1}{C_{*} t^{2}} J_{k, D_{\left(t-t^{2}\right) / 4}}\left(0^{\prime} ; X^{\prime}\right) \leq J_{k, D}\left(-t e_{1} ;\left(0, X^{\prime}\right)\right) \leq \frac{5}{\pi t^{2}} J_{k, D_{\left(t-t^{2}\right) / 4}}\left(0^{\prime} ; X^{\prime}\right)
$$

and

$$
\begin{aligned}
& \frac{1}{C_{*}} \frac{K_{D_{\left(t-t^{2}\right) / 4}}\left(0^{\prime}\right) J_{2, D_{\left(t-t^{2}\right) / 4}}\left(0^{\prime} ; X^{\prime}\right)}{J_{1, D_{\left(t-t^{2}\right) / 4}}\left(0^{\prime} ; X^{\prime}\right)^{2}} \\
& \leq 2-R_{D}\left(-t e_{1} ;\left(0, X^{\prime}\right)\right) \leq C_{*} \frac{K_{D_{\left(t-t^{2}\right) / 4}}\left(0^{\prime}\right) J_{2, D_{\left(t-t^{2}\right) / 4}}\left(0^{\prime} ; X^{\prime}\right)}{J_{1, D_{\left(t-t^{2}\right) / 4}}\left(0^{\prime} ; X^{\prime}\right)^{2}} \text {. }
\end{aligned}
$$

Proof. The fact that $\widetilde{D}_{t} \subset D$ for $0<t<1 / 10$ together with Lemma 2.1 and its corollary gives

$$
\frac{1}{C^{2}} K_{\widetilde{D}_{t}}\left(-t e_{1}\right) \leq K_{D}\left(-t e_{1}\right) \leq K_{\widetilde{D}_{t}}\left(-t e_{1}\right)
$$

and

$$
\begin{aligned}
\frac{1}{C^{2}} J_{k, \widetilde{D}_{t}}\left(-t e_{1} ;\left(0, X^{\prime}\right)\right) & \leq J_{k, D}\left(-t e_{1} ;\left(0, X^{\prime}\right)\right) \\
& \leq J_{k, \widetilde{D}_{t}}\left(-t e_{1} ;\left(0, X^{\prime}\right)\right), \quad k=1,2,
\end{aligned}
$$

for $0<t<1 / 10$ and $X^{\prime} \in \mathbb{C}^{2}$. We use the product formulas

$$
K_{\widetilde{D}_{t}}\left(-t e_{1}\right)=K_{\Delta\left(-t,\left(t-t^{2}\right) / 2\right)}(-t) K_{D_{\left(t-t^{2}\right)} / 4}\left(0^{\prime}\right)
$$

and

$$
J_{k, \widetilde{D}_{t}}\left(-t e_{1} ;\left(0, X^{\prime}\right)\right)=K_{\Delta\left(-t,\left(t-t^{2}\right) / 2\right)}(-t) J_{k, D_{\left(t-t^{2}\right) / 4}}\left(0^{\prime} ; X^{\prime}\right), \quad k=1,2 .
$$

The desired upper and lower bounds now follow from the estimate

$$
\frac{4}{\pi} \frac{1}{t^{2}} \leq K_{\Delta\left(-t,\left(t-t^{2}\right) / 2\right)}(-t)=\frac{4}{\pi} \frac{1}{\left(t-t^{2}\right)^{2}} \leq \frac{5}{\pi t^{2}},
$$

which holds for $0<t<1 / 10$.

Estimate (3) follows from (1) and (2) in conjunction with formula (c), stated at the beginning of this section. This proves Lemma 2.2.

Estimation of the single domain functionals of $D_{s}$ at $0^{\prime}$. For functions $f, g:(0, \alpha) \rightarrow(0, \infty)$ we write $f \approx g$ if there is a constant $C>0$ such that $C^{-1} g(t) \leq f(t) \leq C g(t)$ for all $t \in(0, \alpha)$.

Lemma 2.3. For $1 / 10>s>0$ we have

$$
K_{D_{s}}\left(0^{\prime}\right) \approx \frac{1}{s^{1 / a} \log (1 / s)} .
$$


For $X^{\prime} \in \mathbb{C}^{2} \backslash\{0\}$ we have

$$
\begin{aligned}
& J_{1, D_{s}}\left(0^{\prime} ; X^{\prime}\right) \approx \frac{\left|X^{\prime}\right|^{2}}{s^{1 / m+1 / a}}, \\
& J_{2, D_{s}}\left(0^{\prime} ; X^{\prime}\right) \approx \frac{\left|X^{\prime}\right|^{4}}{s^{2 / m+1 / a}}+\frac{\left|X_{2}\right|^{2}\left|X_{3}\right|^{2}}{s^{2 / a} \log (1 / s)} .
\end{aligned}
$$

Proof. Since $D_{s}$ is a Reinhardt domain, we have

$$
K_{D_{s}}\left(0^{\prime}\right)=\frac{1}{\operatorname{Vol}\left(D_{s}\right)}
$$

as well as

$$
J_{1, D_{s}}\left(0^{\prime} ; X^{\prime}\right)=\sum_{j=2}^{3} \frac{\left|X_{j}\right|^{2}}{\left\|f_{j}\right\|_{D_{s}}^{2}}=\frac{\left|X^{\prime}\right|^{2}}{\left\|f_{2}\right\|_{D_{s}}^{2}},
$$

where $f_{j}\left(z^{\prime}\right):=z_{j}$ for $j=2,3$, and

$$
\begin{aligned}
J_{2, D_{s}}\left(0^{\prime} ; X^{\prime}\right) & =4 \frac{\left|X_{2}\right|^{4}}{\left\|f_{2}^{2}\right\|_{D_{s}}^{2}}+4 \frac{\left|X_{3}\right|^{4}}{\left\|f_{3}^{2}\right\|_{D_{s}}^{2}}+\frac{\left|X_{2}\right|^{2}\left|X_{3}\right|^{2}}{\left\|f_{2} f_{3}\right\|_{D_{s}}^{2}} \\
& =4 \frac{\left|X_{2}\right|^{4}+\left|X_{3}\right|^{4}}{\left\|f_{2}^{2}\right\|_{D_{s}}^{2}}+\frac{\left|X_{2}\right|^{2}\left|X_{3}\right|^{2}}{\left\|f_{2} f_{3}\right\|_{D_{s}}^{2}} .
\end{aligned}
$$

Here we use the symmetry of $D_{s}$.

We have to estimate $\left\|f_{2}^{\nu}\right\|_{D_{s}}^{2}$ for $\nu=0,1,2$ and also $\left\|f_{2} f_{3}\right\|_{D_{s}}^{2}$. Consider the domains

$$
\widehat{D}_{s}:=\left\{z^{\prime} \in \mathbb{C}^{2}|| z_{2}\left|<s^{1 / 2 m},\right| z_{3} \mid<\frac{1}{s^{-1 / 2 m}+s^{-1 / 2 a}\left|z_{2}\right|}\right\} .
$$

Then for $0<s<1 / 10$ one has

$$
\widehat{D}_{s / 4} \subset D_{s} \subset \widehat{D}_{4^{m} s} .
$$

This reduces our task to estimating $\left\|f_{2}^{\nu}\right\|_{\widehat{D}_{\sigma}}^{2}$ for $\nu=0,1,2$ and $\left\|f_{2} f_{3}\right\|_{\widehat{D}_{\sigma}}^{2}$. We have

$$
\begin{aligned}
\left\|f_{2}^{\nu}\right\|_{\widehat{D}_{\sigma}}^{2} & =\int_{\widehat{D}_{\sigma}}\left|z_{2}\right|^{2 \nu} d^{4} z^{\prime}=\pi \int_{\left|z_{2}\right|<\sigma^{1 / 2 m}} \frac{\left|z_{2}\right|^{2 \nu} d^{2} z_{2}}{\left(\sigma^{-1 / 2 m}+\sigma^{-1 / 2 a}\left|z_{2}\right|\right)^{2}} \\
& =2 \pi^{2} \int_{0}^{\sigma^{1 / 2 m}} \frac{r^{2 \nu+1} d r}{\left(\sigma^{-1 / 2 m}+\sigma^{-1 / 2 a} r\right)^{2}} \\
& =2 \pi^{2} \sigma^{1 / m} \int_{0}^{\sigma^{1 / 2 m}} \frac{r^{2 \nu+1} d r}{\left(1+\sigma^{1 / 2 m-1 / 2 a} r\right)^{2}} \\
& =2 \pi^{2} \sigma^{1 / m+(\nu+1)(1 / a-1 / m)} K_{\nu}(\sigma),
\end{aligned}
$$


(substitute $r=: \sigma^{1 / 2 a-1 / 2 m} \varrho$ ) with

$$
\begin{aligned}
K_{\nu}(\sigma) & :=\int_{0}^{\sigma^{1 / m-1 / 2 a}} \frac{\varrho^{2 \nu+1} d \varrho}{(1+\varrho)^{2}}=\int_{0}^{1} \frac{\varrho^{2 \nu+1} d \varrho}{(1+\varrho)^{2}}+\int_{1}^{\sigma^{1 / m-1 / 2 a}} \frac{\varrho^{2 \nu+1} d \varrho}{(1+\varrho)^{2}} \\
& \approx \int_{1}^{\sigma^{1 / m-1 / 2 a}} \frac{\varrho^{2 \nu+1} d \varrho}{(1+\varrho)^{2}} .
\end{aligned}
$$

Note that $\sigma^{1 / m-1 / 2 a}>1$ if $0<\sigma<1$, since $2 a<m$.

But

$$
\int_{1}^{\sigma^{1 / m-1 / 2 a}} \frac{\varrho^{2 \nu+1} d \varrho}{(1+\varrho)^{2}} \approx \int_{1}^{\sigma^{1 / m-1 / 2 a}} \varrho^{2 \nu-1} d \varrho \approx \begin{cases}\sigma^{2 \nu / m-\nu / a} & \text { if } \nu>0 \\ \log (1 / \sigma) & \text { if } \nu=0\end{cases}
$$

Thus we obtain

$$
\left\|f_{2}^{\nu}\right\|_{\widehat{D}_{\sigma}}^{2} \approx \begin{cases}\sigma^{\nu / m+1 / a} & \text { if } \nu \geq 1 \\ \sigma^{1 / a} \log (1 / \sigma) & \text { if } \nu=0\end{cases}
$$

For $\nu=0,1$ this together with $\left(4^{\prime}\right)$ and $\left(5^{\prime}\right)$ implies (4) and (5). If we let $\nu=2$, we find

$$
4 \frac{\left|X_{2}\right|^{4}}{\left\|f_{2}^{2}\right\|_{D_{s}}^{2}}+4 \frac{\left|X_{3}\right|^{4}}{\left\|f_{3}^{2}\right\|_{D_{s}}^{2}} \approx \frac{\left|X^{\prime}\right|^{4}}{s^{2 / m+1 / a}}
$$

Next we check the estimate for the norm $\left\|f_{2} f_{3}\right\|_{\widehat{D}_{\sigma}}$. Similarly to the above we compute

$$
\begin{aligned}
\left\|f_{2} f_{3}\right\|_{\widehat{D}_{\sigma}}^{2} & =\int_{\left|z_{2}\right|<\sigma^{1 / 2 m}}\left|z_{2}\right|^{2}\left(\int_{\left|z_{3}\right|<\left(\sigma^{-1 / 2 m}+\sigma^{-1 / 2 a}\left|z_{2}\right|\right)^{-1}}\left|z_{3}\right|^{2} d^{2} z_{3}\right) d^{2} z_{2} \\
& \left.=2 \pi \int_{\left|z_{2}\right|<\sigma^{1 / 2 m}}\left|z_{2}\right|^{2} \int_{0}^{\left(\sigma^{-1 / 2 m}+\sigma^{-1 / 2 a}\left|z_{2}\right|\right)^{-1}} r^{3} d r\right) d^{2} z_{2} \\
& =\frac{\pi}{2} \int_{\left|z_{2}\right|<\sigma^{1 / 2 m}}\left|z_{2}\right|^{2}\left(\sigma^{-1 / 2 m}+\sigma^{-1 / 2 a}\left|z_{2}\right|\right)^{-4} d^{2} z_{2} \\
& =\pi^{2} \int_{0}^{\sigma^{1 / 2 m}} r^{3}\left(\sigma^{-1 / 2 m}+\sigma^{-1 / 2 a} r\right)^{-4} d r \\
& =\pi^{2} \sigma^{2 / m} \int_{0}^{\sigma^{1 / 2 m}} r^{3}\left(1+\sigma^{1 / 2 m-1 / 2 a} r\right)^{-4} d r \\
& =\pi^{2} \sigma^{2 / a} \int_{0}^{\sigma^{1 / m-1 / 2 a}} \frac{\varrho^{3} d \varrho}{(1+\varrho)^{4}}\left(r=: \sigma^{1 / 2 a-1 / 2 m} \varrho\right)
\end{aligned}
$$




$$
\begin{aligned}
& \approx \sigma^{2 / a} \int_{0}^{\sigma^{1 / m-1 / 2 a}} \frac{\varrho^{3} d \varrho}{1+\varrho^{4}}=\sigma^{2 / a} \int_{0}^{\sigma^{4 / m-2 / a}} \frac{d \varrho^{\prime}}{1+\varrho^{\prime}} \\
& \approx \sigma^{2 / a} \log (1 / \sigma) .
\end{aligned}
$$

This in conjunction with $\left(6^{\prime}\right)$ gives $(6)$, and therefore proves the lemma.

End of the proof of Theorem 1.1. We only need to choose $s=\left(t-t^{2}\right) / 4$ and combine formulas (3) and (4) through (6).

Theorem 1.1 is proved.

\section{References}

[1] S. Bergman, The Kernel Function and Conformal Mapping, Math. Surveys 5, 2nd ed., Amer. Math. Soc., Providence, RI, 1970.

[2] H. Boas, E. Straube and J. Yu, Boundary limits of the Bergman kernel and metric, Michigan Math. J. 42 (1995), 449-461.

[3] D. Catlin, Estimations of invariant metrics in dimension two, Math. Z. 200 (1989), 429-466.

[4] B. Y. Chen and H. Lee, Bergman kernel and complex singularity exponent, preprint, 2006 .

[5] K. Diederich, Über die 1. und 2. Ableitung der Bergmanschen Kernfunktion und ihr Randverhalten, Math. Ann. 203 (1973), 129-170.

[6] K. Diederich and G. Herbort, Pseudoconvex domains of semiregular type, in: Contributions to Complex Analysis and Analytic Geometry, H. Skoda and J. M. Trépreau (eds.), Aspects Math. E 26, Vieweg, Braunschweig, 1994, 127-161.

[7] H. Donnelly, $L^{2}$-cohomology of the Bergman metric for weakly pseudoconvex domains, Illinois J. Math. 41 (1997), 151-160.

[8] S. Fu, Geometry of Reinhardt domains of finite type in $\mathbb{C}^{2}$, J. Geom. Anal. 6 (1996), 407-431.

[9] N. S. Hawley, Constant holomorphic curvature, Canad. J. Math. 5 (1953), 53-56.

[10] G. Herbort, Logarithmic growth of the Bergman kernel for weakly pseudoconvex domains in $\mathbb{C}^{3}$ of finite type, Manuscripta Math. 45 (1983), 69-76.

[11] - On the problem of Kähler convexity in the Bergman metric, Michigan Math. J. 52 (2004), 543-552.

[12] L. Hörmander, $L^{2}$-estimates and existence theorems for the $\bar{\partial}$-operator, Acta Math. 113 (1965), 89-152.

[13] -, An Introduction to Complex Analysis in Several Variables, 3rd ed., NorthHolland Math. Library 7, North-Holland, 1990.

[14] K. T. Kim and J. Yu, Boundary behavior of the Bergman curvature in strictly pseudoconvex polyhedral domains, Pacific J. Math. 176 (1996), 141-163.

[15] P. Klembeck, Kähler metrics of negative curvature, the Bergmann metric near the boundary, and the Kobayashi metric on smooth bounded strictly pseudoconvex sets, Indiana Univ. Math. J. 27 (1978), 275-282.

[16] S. G. Krantz and J. Yu, On the Bergman invariant and curvatures of the Bergman metric, Illinois J. Math. 40 (1996), 226-244.

[17] Q. K. Lu, On Kähler manifolds with constant curvature, Acta Math. Sinica 16 (1966), 269-281 (in Chinese); English transl.: Chinese Math. Acta 8 (1966), 283298. 
[18] J. D. McNeal, Holomorphic sectional curvature of some pseudoconvex domains, Proc. Amer. Math. Soc. 107 (1989), 113-117.

[19] J. Yu, Peak functions on weakly pseudoconvex domains, Indiana Univ. Math. J. 43 (1994), 1271-1295.

Bergische Universität Wuppertal

Fachbereich C - Mathematik und Naturwissenschaften

Gaußstraße 20

D-42097 Wuppertal, Germany

E-mail: gregor@math.uni-wuppertal.de

Received 23.11.2006

and in final form 22.1.2007 